\title{
GABA Concentrations in the Anterior Cingulate Cortex Are Associated with Fear Network Function and Fear Recovery in Humans
}

\author{
Nina Levar ${ }^{1,2,3,4 *}$, Judith M. C. van Leeuwen ${ }^{1,5}$, Nicolaas A. J. Puts ${ }^{6,7}$, Damiaan Denys 1, 2,3,4 $^{2}$ \\ and Guido A. van Wingen ${ }^{1,2,3,4}$ \\ ${ }^{1}$ Department of Psychiatry, Academic Medical Center, Amsterdam, Netherlands, ${ }^{2}$ Brain Imaging Center, Academic Medical \\ Center, Amsterdam, Netherlands, ${ }^{3}$ Amsterdam Brain and Cognition, University of Amsterdam, Amsterdam, Netherlands, \\ ${ }^{4}$ Spinoza Center for Neuroimaging, Amsterdam, Netherlands, ${ }^{5}$ Department of Psychiatry, University Medical Center Utrecht, \\ Utrecht, Netherlands, ${ }^{6}$ Russell H. Morgan Department of Radiology and Radiological Science, Johns Hopkins University, \\ Baltimore, MD, USA, ${ }^{7}$ FM Kirby Center for Functional Brain Imaging, Kennedy Krieger Institute, Baltimore, MD, USA
}

\section{OPEN ACCESS}

Edited by:

Joshua Oon Soo Goh,

National Taiwan University, Taiwan

Reviewed by:

Shih-Wei Wu,

National Yang-Ming University, Taiwan

Stefano Delli Pizzi,

University of Chieti-Pescara, Italy

Ming-Tsung Tseng,

National Taiwan University, Taiwan

*Correspondence:

Nina Levar

levar.nina@gmail.com

Received: 20 September 2016 Accepted: 07 April 2017 Published: 27 April 2017

Citation:

Levar N, van Leeuwen JMC

Puts NAJ, Denys $D$ and van Wingen GA (2017) GABA

Concentrations in the Anterior Cingulate Cortex Are Associated with

Fear Network Function and Fear

Recovery in Humans.

Front. Hum. Neurosci. 11:202.

doi: 10.3389/fnhum.2017.00202
Relapse of fear after successful treatment is a common phenomenon in patients with anxiety disorders. Animal research suggests that the inhibitory neurotransmitter $\gamma$-aminobutyric acid (GABA) plays a key role in the maintenance of extinguished fear. Here, we combined magnetic resonance spectroscopy and functional magnetic resonance imaging to investigate the role of GABA in fear recovery in 70 healthy male participants. We associated baseline GABA levels in the dorsal anterior cingulate cortex (dACC) to indices of fear recovery as defined by changes in skin conductance responses (SCRs), blood oxygen level dependent responses, and functional connectivity from fear extinction to fear retrieval. The results showed that high GABA levels were associated with increased SCRs, enhanced activation of the right amygdala, and reduced amygdala-ventromedial prefrontal cortex connectivity during fear recovery. Follow-up analyses exclusively for the extinction phase showed that high GABA levels were associated with reduced amygdala activation and enhanced amygdala-ventromedial prefrontal cortex connectivity, despite the absence of correlations between GABA and physiological responses. Follow-up analyses for the retrieval phase did not show any significant associations with GABA. Together, the association between GABA and increases in SCRs from extinction to retrieval, without associations during both phases separately, suggests that dACC GABA primarily inhibits the consolidation of fear extinction. In addition, the opposite effects of GABA on amygdala activity and connectivity during fear extinction compared to fear recovery suggest that dACC GABA may initially facilitate extinction learning.

Keywords: fear, extinction, recovery, fMRI, MRS, GABA, SCR, dACC

\section{INTRODUCTION}

The experience of a dangerous or harmful situation often results in the implicit association of a neutral stimulus with the fear experience. This stimulus can then elicit a fear response, even in the absence of the potentially aversive outcome, which is seen in patients with anxiety disorders. Exposure therapy makes use of extinction processes and is aimed at overcoming these inappropriate 
responses by repeatedly exposing individuals to the feared stimulus, gradually resulting in a new safe association which inhibits the old fear memory (Bouton, 2004). Despite shortterm benefits of exposure therapy, relapse into fear is a common problem in the treatment of anxiety disorders such as posttraumatic stress disorder (PTSD) (Durham et al., 2005; Milad et al., 2008; Duits et al., 2015). This phenomenon is known as the spontaneous recovery of fear and is highly variable between individuals (Pavlov, 1927; Milad and Quirk, 2002; Bush et al., 2007).

Theoretical accounts posit that spontaneous recovery is the consequence of reduced inhibition of conditioned responses (Rescorla, 2004), and pharmacological studies in rodents point to a key role of the primary inhibitory neurotransmitter $\gamma$ aminobutyric acid (GABA) in the acquisition and maintenance of fear inhibition. These studies indicate that increased GABAergic neurotransmission during extinction training initially reduces fear expression. In contrast, increasing GABAergic neurotransmission disrupts the consolidation of the acquired extinction memory which consequently leads to stronger recovery of fear (Bindra et al., 1965; Bouton et al., 1990; McGaugh et al., 1990; Makkar et al., 2010).

Despite growing insight into the neurocircuitry of fear related processes, the underlying neurochemical basis of human fear learning is largely unknown. While rodent studies have investigated manipulations of GABAergic neurotransmission using high pharmacological doses, little is known about the role of natural variations in endogenous GABA levels. Proton magnetic resonance spectroscopy $\left({ }^{1} \mathrm{H}-\mathrm{MRS}\right)$ enables us to noninvasively assess brain metabolite concentrations in vivo and to relate these to individual differences in BOLD responses and cognitive processes (Northoff et al., 2007; Muthukumaraswamy et al., 2009; Sumner et al., 2010). A recent study pointed to a dysregulation of prefrontal GABA in anxiety disorders by showing that GABA levels in the medial prefrontal cortex (mPFC) in PTSD patients are increased (Michels et al., 2014). In line with this, Delli Pizzi et al. (2016b) showed that GABA levels in the vmPFC were positively correlated with anxiety as scored by the State Trait Anxiety Inventory (STAI-Y2) in healthy individuals.

In humans, the dorsal anterior cingulate cortex (dACC) as part of the mPFC has been shown to play a key role in the reappraisal of negative emotions and the expression of fear during fear conditioning (Milad et al., 2007; Sehlmeyer et al., 2009; Etkin et al., 2011). The dACC, ventromedial (vmPFC), and amygdala are tightly interconnected and compose a core circuit within a comprehensive fear network (Phelps et al., 2004; Ghashghaei et al., 2007; Milad et al., 2007; Shin and Liberzon, 2010; Klavir et al., 2013). Amygdala reactivity has been shown to covary with individual differences in fear responses and conditionability, rather than being representing fear responses in general (Bach et al., 2011; MacNamara et al., 2015). The vmPFC is primarily linked to extinction learning and the later retrieval of extinction and has been indicated to play a role in extinction learning with a proposed regulatory function of fear responses by exerting a modulatory impact on the amygdala (Quirk et al., 2003; Rosenkranz et al., 2003; Motzkin et al., 2015). The interplay between the amygdala and both $\mathrm{mPFC}$ and $\mathrm{dACC}$ has been indicated in memory consolidation following a fear conditioning and extinction paradigm (Feng et al., 2014). Resting fMRI revealed enhanced amygdaladACC coupling, and decreased amygdala-mPFC coupling during memory consolidation following fear learning and extinction. Furthermore, a recent study showed that resting activity in the amygdala and the vmPFC were anti-correlated, with both trait anxiety scores and mPFC GABA levels being inversely correlated with amygdala-vmPFC coupling (Delli Pizzi et al., 2016a).

In the present study, we investigated associations of individual GABA concentrations in the dACC of healthy male volunteers with the recovery of fear, and assessed how GABA levels contribute to the consolidation and retrieval of previously extinguished fear. We focused on the influence of GABA on three core structures of the fear network: the amygdala, the vmPFC, and the dACC. On the basis of the above outlined work in rodents, we hypothesized that high GABA concentrations would initially facilitate extinction learning while subsequently hamper the consolidation of extinction learning, which is expected to result in heightened recovery of previously extinguished fear.

\section{MATERIALS AND METHODS}

\section{Participants}

To investigate the impact of GABA on the recovery of fear, 106 healthy right-handed men between the ages of 18-35 years $(M=21.75$ years, $S D=3.17)$ participated in a 2-day multi-modal MRI study (Figure 1; Supplemental Information for details). Data were unavailable or excluded due to one or more of the following reasons: technical problems (nine missing data sets), poor quality of the MRS spectra (27; see below), and skin conductance response (SCR) related technical issues during recordings (day 1: fifteen; day 2: seventeen). As extinction learning and extinction memory retrieval is dependent on successful conditioning procedures, six participants were excluded from the analysis as they showed an average SCR in the last four trials of the conditioning phase below 2 standard deviations of the group mean. Accordingly, data of 70 subjects were complete for inclusion in fMRI-MRS correlation analyses, and data of 57 subjects were complete for inclusion in the SCRMRS correlation analyses. Informed consent was obtained before entering the study. The study was performed in accordance with the declaration of Helsinki and approved by the Medical Ethics Committee of the Academic Medical Center in Amsterdam.

\section{Procedures}

Participants completed a 2-day fear conditioning protocol in a $3 \mathrm{~T}$ whole-body MRI scanner (Philips, 3.0T Achieva) equipped with a 32-channel SENSE head-coil. On day one, a ${ }^{1} \mathrm{H}$ MEGA-PRESS (Mescher et al., 1998; Waddell et al., 2007) scan of the dACC was acquired before undergoing a fear conditioning and extinction session during functional MRI (for details on acquisition parameters, see Supplemental Information). Approximately $24 \mathrm{~h}$ later, participants returned to test extinction retrieval during functional MRI. During the conditioning paradigm, mild electrical stimulation to the participants' right wrist was 


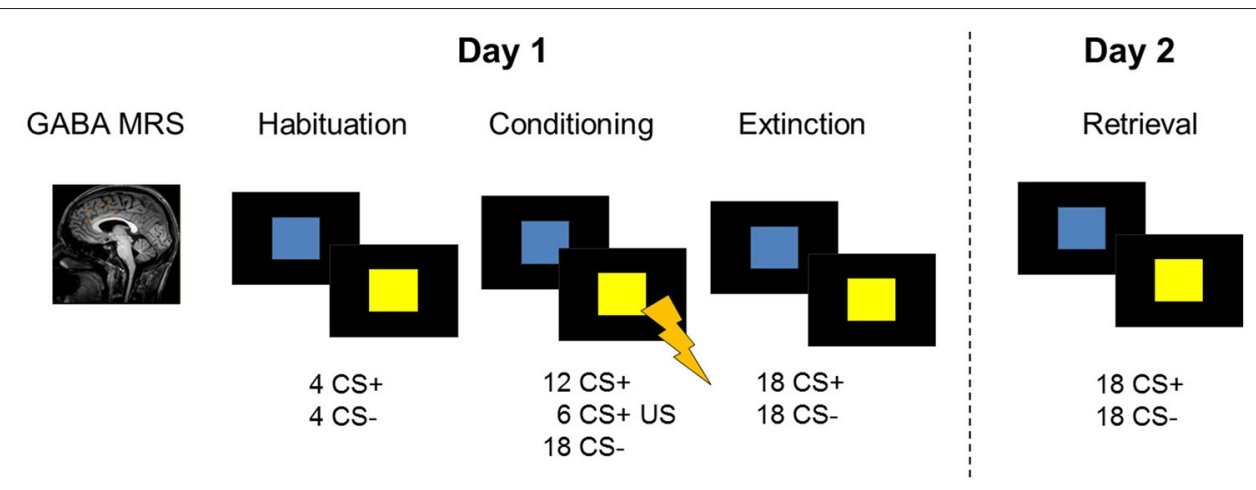

FIGURE 1 | Description of the 2-day study design including GABA MRS of the dACC and a fear conditioning and extinction paradigm during fMRI on experimental day 1. On the following day, participants were tested for memory retrieval during fMRI. Skin conductance responses were recorded as a psychophysiological measure of on both days.

used as unconditioned stimulus. Individual stimulation intensity settings were determined prior to the experimental session by ramping up the current in small steps ( $2 \mathrm{~mA})$ until the participant described the sensation as "highly annoying but not painful." Skin conductance responses were recorded as a psychophysiological measure of fear throughout the functional MRI sessions.

\section{Fear Conditioning and Extinction Task}

The fear conditioning task included two conditioned stimuli (CSs) which consisted of a blue and a yellow square. On the first experimental day, the paradigm started with a habituation phase ( 8 trials) and was followed by the conditioning phase (36 trials) during which one stimulus (CS+) was paired with an unconditioned aversive stimulus (US) in $33 \%$ of the trials. The unconditioned stimulus consisted of a mild electric shock to the participant's right wrist and was administered at stimulus offset. US intensities were determined before the start of the scan in order to ascertain that the electric stimulus was unpleasant but not painful (see Supplemental Information). The other stimulus (CS-) was never paired with the US. The color of the CS+ was counterbalanced across subjects. Stimuli were presented for $4 \mathrm{~s}$ with an inter-stimulus interval ranging between 6.5 and 9.5 s. Shortly (20 s) after the conditioning phase, a fear extinction training was performed. During the extinction phase (36 trials), all conditioned stimuli were shown in the absence of the US. Instructions were presented on screen before every task phase, explaining that colored squares will be presented on screen and that shocks may be administered during the task. Further instructions stated that there is a relationship between the squares and the shock. On the second experimental day, SCR recordings were prepared as on day 1 and stimulation intensities were determined again. Extinction retrieval was tested by presenting the CSs in the absence of the unconditioned stimulus during fMRI (24 trials).

\section{Skin Conductance Responses}

Skin conductance data were analyzed using NetStation software (Electrical Geodesics, Inc., Oregon, United States). A NetStation filter was used to remove large artifacts resulting from MR signals using a 2 TR window. Continuous SCR data was further analyzed using Matlab (Matlab Release 2014a, The MathWorks, Inc., Natick, Massachusetts, United States) and EEGLAB for Matlab (Delorme and Makeig, 2004). A median filter and a low pass filter were applied to remove remaining MR artifacts. SCR changes in response to stimulus presentation were quantified using the minimum response between stimulus onset and $2 \mathrm{~s}$ post-stimulus onset to accommodate the ongoing decrease in SCR to the previous stimulus. The maximum response between stimulus onset and $8 \mathrm{~s}$ post-stimulus was extracted to calculate the change in SCR to the stimulus. Due to large inter-individual differences in SCRs, data was standardized to the largest elicited SCRs ( $\max (\mathrm{SCR})$ ), which were the unconditioned responses to the shocks. We used the average of the SCRs (Avgmax(SCR) to the US responses to standardize data (SCR/Avg $\max (\mathrm{SCR})$ ). In order to investigate the recovery of fear, differences in fear responses between the two experimental days were calculated. Including SCRs to both the extinguished stimuli on day 1 and responses to the retrieved stimulus on day 2 , enabled us to take individual differences in fear extinction into account, as SCRs to the CSs varied between participants at the end of extinction learning. As there is no gold standard, a fear recovery index for skin conductance responses was defined for each participant as the differential response to the first trial during extinction retrieval (when fear recovery is maximal followed by rapid re-extinction) and the mean response to the last two trials during extinction training, to increase signal to noise by averaging over maximally extinguished trials. SPSS (IBM Corp. Released 2011. IBM SPSS Statistics for Windows, Version 20.0. Armonk, NY: IBM Corp) was used for statistical testing. Non-parametric tests (WilcoxonMann-Whitney test) were used to assess differences in skin conductance responses due to CS types within and between phases. Non-parametric correlation analyses (Spearman's) were performed between GABA concentrations and SCRs of fear recovery, extinction retrieval, and extinction.

\section{MRS}

GABA-edited MRS spectra were acquired from a $40 \times 20 \times$ $20 \mathrm{~mm}^{3}$ volume placed in the dorsal anterior cingulate cortex. The voxel was placed and tilted for the lower long side to be 
closely aligned with the corpus callosum (Figure 2A). GABA MRS spectra were acquired using a MEGA-PRESS sequence with the following parameters: TR/TE $=2,000 / 73,384$ averages, $\mathrm{T}_{\text {Acq }}=12: 48$ min (Mescher et al., 1998; Waddell et al., 2007). Spectra were analyzed using AMARES in jMRUI, a non-linear -least-squares quantitation algorithm (Naressi et al., 2001). In a first step, any residual water signal was removed using the Hankel Lanczos singular value decomposition (HLSVD) filter. Pre-processing of both unedited and difference spectra included apodization (Lorentzian $5 \mathrm{~Hz}$ ), zero filling $(1,024)$, and zerothorder phase correction with respect to NAA. Difference spectra were phased at $180^{\circ}$. GABA+ (GABA and macro-molecules) was fitted onto the difference spectra at $3.0 \mathrm{ppm}$ using a single Gaussian function (Figure 2B). Unedited spectra were fitted for NAA (2.0 ppm), Cr+ (3.0 ppm), and Cho (3.2 ppm) using a Lorentzian curve. Creatine was used as an internal standard for GABA+ quantification. All spectra were independently rated by two of the researchers and 27 spectra were excluded from further analysis due to poor quality. Both difference and unedited spectra were excluded if the line width of the NAA peak exceeded $11 \mathrm{~Hz}$. Importantly, the MRS quality control was performed independently from the subsequent analyses.

\section{MRI Data Analysis}

Functional MRI data were analyzed using the Statistical Parametric Mapping toolbox (SPM8; Wellcome Trust Center for Neuroimaging, London, UK). Standard pre-processing steps were applied including realignment, slice time correction, coregistration to structural images, normalization into Montreal Neurological Image template space using the segmentation procedure, and spatial smoothing ( $8 \mathrm{~mm}$ FMWH). Data of the two experimental days were analyzed separately using a linear convolution model of the canonical hemodynamic response function. Low frequency drift was removed using a high pass filter $(1 / 128 \mathrm{~Hz})$. A design matrix which involved separate regressors for $\mathrm{CS}+$ and $\mathrm{CS}-$ trials for each early (i.e., the first $6 \mathrm{CS}+$ and CS- trials) and late phases (i.e., the last six CS+ and CS - trials) of habituation, conditioning, and extinction for data of day one was defined for each participant. For the conditioning phase, reinforced and non-reinforced CS + trials were modeled separately. For further analyses, only the nonreinforced of all $\mathrm{CS}+$ trials were analyzed to avoid measuring BOLD responses to the US. Data of the second experimental session involved experimental conditions for early and late CS+ and CS - trials of the retrieval phase. The six parameters obtained from the realignment procedure were added as regressors of no interest into the design matrix. The obtained contrast images were then entered into a second level full-factorial design in order to assess task effects. A voxel-wise correlation analysis was performed to test for correlations between GABA concentrations and BOLD responses. GABA+/Cr+ ratios were added as covariates to the full-factorial design including $\mathrm{CS}+$ and $\mathrm{CS}-$ responses during the late phase of extinction and the early phase of extinction retrieval. To assess the recovery of fear, the differential response $(\mathrm{CS}+>\mathrm{CS})$ during the early phase of retrieval was compared to the differential response during the late phase of extinction (early retrieval ${ }_{(\mathrm{CS}+}>\mathrm{CS}-$ ) $>$ late extinction $(\mathrm{CS}+>\mathrm{CS}-)$ ). To investigate functional coupling between brain regions, we used a generalized psychophysiological interaction analysis (gPPI) to assess connectivity of the right amygdala with all other brain voxels (http://brainmap.wisc.edu/PPI; McLaren et al., 2012). Generalized PPI analysis allows for the assessment of functional connectivity and interactions between different brain regions in a task-dependent manner by incorporating
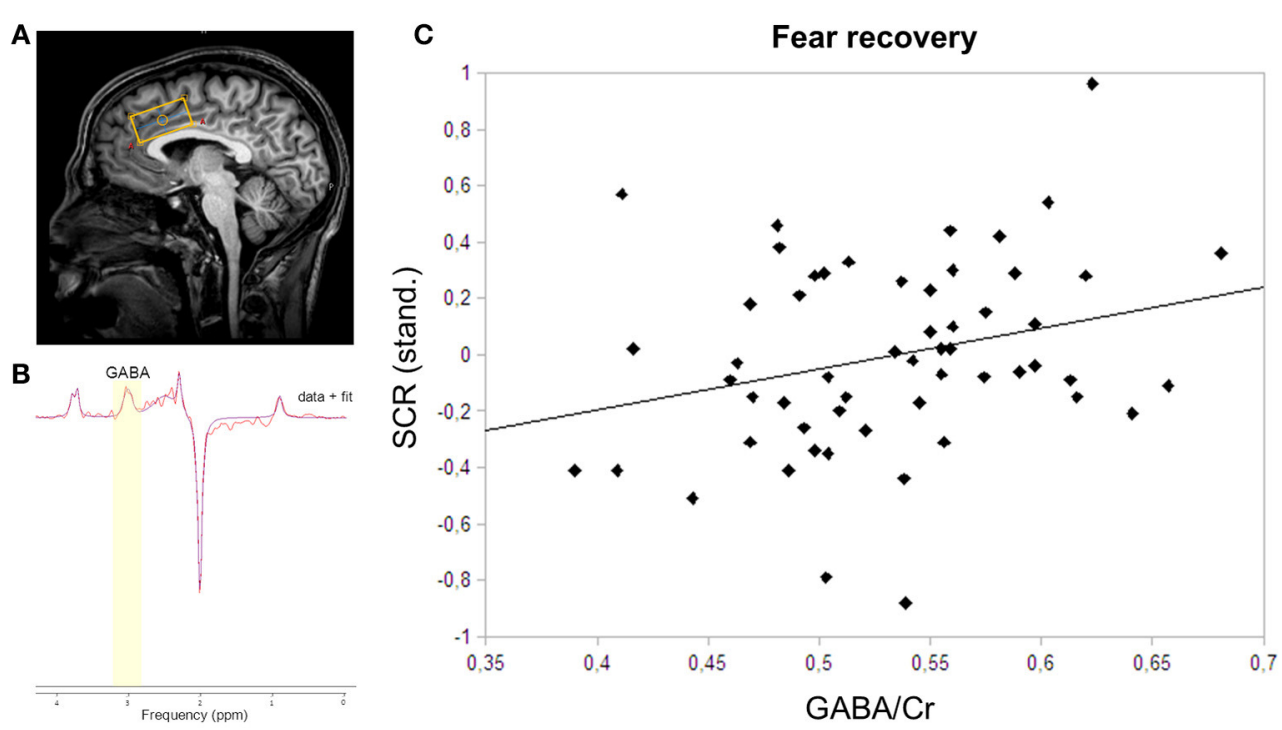

FIGURE 2 | MRS voxel placement, MRS spectrum with fit, and correlation plot of GABA and skin conductance responses. (A) The MRS voxel was planned on the anatomical scan of each participant. The $40 \times 20 \times 20 \mathrm{~mm}^{3}$ box was closely aligned with the corpus callosum in the dACC. (B) GABA was fitted onto the spectrum using AMARES in jMRUI. GABA was fitted using a Gaussian function at 3.0 ppm. (C) The correlation of dACC GABA concentrations and skin conductance responses to the CS + relative to the CS- during fear recovery revealed a positive relation $\left(r_{S}=0.28 ; P=0.04\right)$. 
multiple contrasts. We included our contrasts of interest (i.e., retrieval and extinction: $\mathrm{CS}+>\mathrm{CS}-$ ) and used GABA levels as covariates to examine correlations between $\mathrm{AACC}$ GABA and functional connectivity. The amygdala seed was defined as a 5 $\mathrm{mm}$ sphere around the group peak activity of GABA modulated fear recovery within the amygdala obtained from second level analysis.

All statistical tests were family-wise error (FWE) rate corrected for multiple comparisons across the whole brain on cluster level $(p<0.05)$ with an initial height threshold of $p<0.001$ (Eklund et al., 2016), and statistical tests for the three regions of interest (dACC, amygdala, and vmPFC) were FWE rate corrected $(p<0.05)$ on voxel level using small-volume corrections (Worsley et al., 1996). The dACC was defined as the dACC MRS voxel, the amygdala was anatomically defined using the Automated Anatomical Labeling atlas (Tzourio-Mazoyer et al., 2002), and the vmPFC (sphere with $10 \mathrm{~mm}$ radius centered at $\mathrm{MNI}_{\mathrm{x}, \mathrm{y}, \mathrm{z}}=-2,52,-2$ ) was defined similarly to the vmPFC previously implicated in studies of fear extinction and fear recall in healthy humans (see Milad et al., 2013).

\section{RESULTS}

\section{Psychophysiology}

To assess the recovery of previously extinguished fear, we first assured successful conditioning to the CS+ on the physiological level. Skin conductance responses differed significantly between $\mathrm{CS}+$ and $\mathrm{CS}-$ in the late phase of conditioning $(P<0.0001)$, thereby indicating successful acquisition of a fear memory to the conditioned stimulus (Table 1). During extinction training, participants showed a strong decrease in SCRs (late extinction vs. late conditioning for $\mathrm{CS}+>\mathrm{CS}-; P<0.0001)$. During extinction retrieval, responses to the CS+ were significantly higher than to the CS $-(P=0.034)$. Skin conductance responses revealed a recovery of fear (i.e., the change in fear responses to the conditioned stimuli from the last two trials of extinction learning to the first response to the non-reinforced stimulus on the following day) to both the CS+ $(P<0.001)$ and the CS- $(P<0.001)$, suggesting that fear recovery generalized to both conditions across all participants. To assess individual differences in fear recovery that are specific to the $\mathrm{CS}+$, an index of fear recovery was calculated for each participant (first trial extinction retrieval $>$ last two trials of extinction for $\mathrm{CS}+>$ CS-). Further supporting a recovery of fear, skin conductance responses to the CS+ were significantly higher during extinction retrieval than during habituation $(P=0.015)$, but not to the CS- $(P=0.204)$. To test our hypothesis of the involvement of GABA in the recovery of extinguished fear, a non-parametric correlation analysis showed that individuals with high GABA levels experienced stronger fear recovery $\left(r_{s}=0.28 ; P=0.035\right.$; Figure 2C). Further exploration did not show an association between GABA levels and fear responses during the extinction or the retrieval phase separately (extinction: $r_{s}=-0.18 ; P=$ 0.181 ; retrieval: $r_{s}=0.14 ; P=0.311$ ), suggesting that GABA mainly mediates the consolidation of the extinction memory from extinction training to extinction retrieval.
TABLE 1 | Skin conductance responses.

\begin{tabular}{lll}
\hline Fear learning phase & CS $+($ mean + SD) & CS $-($ mean + SD) \\
\hline Habituation & $0.499 \pm 0.312$ & $0.499 \pm 0.302$ \\
Early acquisition & $0.844 \pm 0.357$ & $0.769 \pm 0.336$ \\
Late acquisition & $0.593 \pm 0.304$ & $0.282 \pm 0.223$ \\
Early extinction & $0.580 \pm 0.334$ & $0.497 \pm 0.31$ \\
Late extinction & $0.263 \pm 0.304$ & $0.179 \pm 0.212$ \\
Early retrieval & $0.521 \pm 0.337$ & $0.443 \pm 0.317$ \\
\hline
\end{tabular}

Means and standard deviations of the standardized skin conductance responses averaged over all participants for habituation, early (first two trials) and late phases (last two trials) of fear acquisition and fear extinction, and early extinction retrieval (one trial).

\section{Functional MRI}

Functional MRI data analysis confirmed the successful acquisition of fear. For all contrasts, activation to the CS+ in relation to the $\mathrm{CS}-$ is reported, and the results are corrected for multiple voxel-wise comparisons $(P<0.05$; see methods). During the conditioning phase, activation in the fear related network including the middle cingulate gyrus, right insular lobe, right and left inferior frontal gyrus, right and left supramarginal gyri, left pallidum, left thalamus, right middle frontal gyrus, right and left precentral gyri, and the left amygdala was observed. To reveal the neural basis underlying the recovery of fear, we first assessed the brain regions involved in fear recovery. This analysis showed increased BOLD responses in regions within the fear network including the thalamus, the left post-central gyrus, the left inferior frontal and parietal gyrus, the right supramarginal gyrus, and the right rolandic perculum (Figure 3A, Table 2). To test our hypothesis of the involvement of GABA in the recovery of extinguished fear on the neural level, we included individual GABA concentrations in a voxel-wise correlation analysis of our fMRI data in order to investigate whether differences in activation in the fear network during fear recovery were mediated by individual differences in dACC GABA concentrations. This analysis showed that individuals with high levels of GABA showed a stronger recovery of previously extinguished fear in the right amygdala than participants with low dACC GABA levels (Figures 3B,C, Table 3). We subsequently analyzed the association between GABA and BOLD responses for the extinction learning and retrieval phases separately (Figure 4A). BOLD activity during the extinction retrieval phase was not significantly correlated with GABA levels. In contrast, we did observe a significant negative correlation between GABA concentrations and BOLD responses in the right amygdala, the cerebellum, the middle cingulate gyrus, and the right insula during the late phase of extinction learning (Figure 4, Table 3). No significant correlations were observed between SCR measures and BOLD activity during extinction, retrieval, and fear recovery.

\section{Functional Connectivity}

The functional MRI results reported above showed a positive correlation between GABA concentrations in the dACC and activity in the right amygdala during the recovery of fear. To explain the functional interplay between these two regions, we aimed to investigate functional connectivity in order to examine 
TABLE 2 | Group activation for fear recovery, late extinction and early extinction retrieval.

\begin{tabular}{|c|c|c|c|c|}
\hline Area of activation & $P$-value FWE cluster-level & Cluster size \# of voxels & $\mathbf{T}$ (peak) & Peak voxel MNI coordinates \\
\hline \multicolumn{5}{|c|}{ FEAR RECOVERY: RETRIEVAL EARLY > EXTINCTION LATE } \\
\hline \multicolumn{5}{|l|}{$\mathrm{CS}_{+}>\mathrm{CS}-$} \\
\hline Thalamus & $<0.0001$ & 573 & 5.41 & $0,-28,-2$ \\
\hline Left post-central gyrus & 0.002 & 453 & 4.65 & $-48,-20,24$ \\
\hline Left inferior parietal lobule & & & 3.52 & $-54,-28,42$ \\
\hline Left inferior frontal gyrus & 0.026 & 247 & 4.54 & $-48,6,12$ \\
\hline Right supramarginal gyrus & 0.017 & 277 & 4.51 & $44,-30,24$ \\
\hline Right rolandic operculum & & & 4.07 & $38,-34,20$ \\
\hline Right rolandic operculum & 0.048 & 207 & 4.41 & $46,2,12$ \\
\hline \multicolumn{5}{|l|}{ EARLY RETRIEVAL } \\
\hline \multicolumn{5}{|l|}{$\mathrm{CS}_{+}>\mathrm{CS}_{-}$} \\
\hline Right insula lobe & $<0.0001$ & 15034 & 8.62 & $34,22,-4$ \\
\hline Left insula lobe & & & 8.36 & $-30,24,-6$ \\
\hline Left middle cingulate cortex & & & 8.18 & $-46,2,310$ \\
\hline Left middle cingulate cortex & $<0.0001$ & 7009 & 8.18 & $-4,8,38$ \\
\hline Right middle cingulate cortex & & & 7.90 & $6,4,36$ \\
\hline Left SMA & & & 6.64 & $-2,-2,58$ \\
\hline Right supramarginal gyrus & $<0.0001$ & 1513 & 6.47 & $56,-28,28$ \\
\hline Right supramarginal gyrus & & & 6.31 & $64,-28,30$ \\
\hline Inferior parietal cortex & & & 5.16 & $46,-36,20$ \\
\hline Right cerebellum & 0.018 & 270 & 5.04 & $36,-48,-32$ \\
\hline Right cerebellum & & & 3.74 & $24,-54,-24$ \\
\hline Right precentral gyrus & 0.044 & 211 & 4.61 & $48,4,38$ \\
\hline Right precentral gyrus & & & 3.50 & $36,0,42$ \\
\hline \multicolumn{5}{|l|}{$\mathrm{CS}_{-}>\mathrm{CS}+$} \\
\hline Right paracentral lobule & 0.017 & 274 & 6.42 & $8,-26,62$ \\
\hline Left paracentral lobule & & & 5.80 & $-6,-32,62$ \\
\hline Left inferior temporal gyrus & 0.001 & 456 & 5.94 & $-56,-6,-28$ \\
\hline Left medial temporal pole & & & 4.17 & $-48,14,-34$ \\
\hline Left middle temporal gyrus & & & 5.09 & $-60,-14,-20$ \\
\hline Right angular gyrus & $<0.0001$ & 630 & 5.91 & $52,-60,26$ \\
\hline Right angular gyrus & & & 3.25 & $42,-64,48$ \\
\hline Left superior medial gyrus & $<0.0001$ & 1530 & 5.41 & $-8,54,28$ \\
\hline Right superior frontal gyrus & & & 4.80 & $22,62,10$ \\
\hline Left superior medial gyrus & & & 4.64 & $-10,46,44$ \\
\hline Right precuneus & $<0.0001$ & 1355 & 5.35 & $6,-54,26$ \\
\hline Left middle cingulate cortex & & & 4.53 & $-14,-48,32$ \\
\hline Right precuneus & & & 4.42 & $12,-48,12$ \\
\hline Left precentral gyrus & 0.025 & 247 & 4.83 & $56,-4,28$ \\
\hline Right post-central gyrus & & & 3.69 & $42,-8,28$ \\
\hline Left angular gyrus & $<0.0001$ & 580 & 4.52 & $-52,-64,24$ \\
\hline Left angular gyrus & & & 4.32 & $-44,-62,26$ \\
\hline Left angular gyrus & & & 4.31 & $-42,-52,24$ \\
\hline Right post-central gyrus & 0.005 & 354 & 4.47 & $36,-26,48$ \\
\hline Right precentral gyrus & & & 4.08 & $34,-18,60$ \\
\hline Right precentral gyrus & & & 3.72 & $32,-26,58$ \\
\hline Right superior frontal gyrus & $<0.0001$ & 694 & 4.37 & $20,34,52$ \\
\hline \multicolumn{5}{|l|}{ LATE EXTINCTION } \\
\hline \multicolumn{5}{|l|}{$\mathrm{CS}_{+}>\mathrm{CS}_{-}$} \\
\hline Right insula lobe & $<0.0001$ & 1890 & 7.96 & $32,24,-4$ \\
\hline Left insula lobe & $<0.0001$ & 1193 & 6.13 & $-32,22,-8$ \\
\hline
\end{tabular}


TABLE 2 | Continued

\begin{tabular}{|c|c|c|c|c|}
\hline Area of activation & $P$-value FWE cluster-level & Cluster size \# of voxels & $\mathbf{T}$ (peak) & Peak voxel MNI coordinates \\
\hline \multicolumn{5}{|l|}{ LATE EXTINCTION } \\
\hline \multicolumn{5}{|l|}{$\mathrm{CS}_{+}>\mathrm{CS}_{-}$} \\
\hline Left superior temporal gyrus & & & 3.65 & $-48,6,-6$ \\
\hline Right superior medial gyrus & $<0.0001$ & 2133 & 5.09 & $6,26,48$ \\
\hline Right supramarginal gyrus & 0.019 & 292 & 4.37 & $60,-40,30$ \\
\hline \multicolumn{5}{|l|}{$\mathrm{CS}_{-}>\mathrm{CS}_{+}$} \\
\hline Right angular gyrus & $<0.0001$ & 5355 & 6.22 & $52,-64,24$ \\
\hline Left angular gyrus & & & 6.16 & $-46,-72,28$ \\
\hline Right middle frontal gyrus & 0.032 & 255 & 4.87 & $28,24,42$ \\
\hline Right middle frontal gyrus & & & 3.27 & $30,22,54$ \\
\hline Right parahippocampal gyrus & 0.003 & 448 & 4.82 & $28,-34,-8$ \\
\hline Right parahippocampal gyrus & & & 4.38 & $30,-30,-16$ \\
\hline Right parahippocampal gyrus & & & 4.11 & $34,-40,-8$ \\
\hline Left hippocampus & 0.003 & 302 & 4.72 & $-24,-20,-20$ \\
\hline Left cerebellum & & & 3.95 & $-20,-30,-26$ \\
\hline Left hippocampus & & & 3.63 & $-20,-8,-22$ \\
\hline Right precentral gyrus & 0.006 & 381 & 3.84 & $38,-24,62$ \\
\hline Right precentral gyrus & & & 3.83 & $38,-22,42$ \\
\hline
\end{tabular}

All results are $P<0.05$ cluster-level FWE corrected with an initial height threshold of $P=0.001$, uncorrected.

${ }^{*}$ Small volume corrected with $P_{\text {peak voxel }}<0.05$.

whether and to what extent this connectivity may be mediated by dACC GABA concentrations. As GABA concentrations in the dorsal ACC correlated with BOLD signal in the right amygdala, we further explored potential interrelations between these core regions and the vmPFC as a modulatory interconnected region within this circuit. A psychophysiological interaction analysis revealed a negative correlation of dACC GABA and functional connectivity between the vmPFC and the right amygdala during fear recovery (Retrieval ${ }_{(\mathrm{CS}+}>\mathrm{CS}-$ ) $>$ Extinction $\left._{(\mathrm{CS}>\mathrm{CS}-)}\right)$. Reduced connectivity to the CS+ (relative to the CS-) during extinction learning compared to extinction retrieval suggests that high dACC GABA levels may lead to reduced inhibitory control of the vmPFC on amygdala function, and thereby result in increased fear recovery (Figure 5, Table 3). To disentangle contributions of the associated phases, we separately examined connectivity for the early retrieval and the late extinction phases. In contrast to the negative GABAergic impact during fear recovery, the results showed a positive association between dACC GABA on amygdala-vmPFC coupling separately during late extinction during $\mathrm{CS}+$ trials relative to CS- (Figure 6, Table 3). During the retrieval phase, GABA did not mediate connectivity of the amygdala and any other region.

\section{DISCUSSION}

This study reveals that the main inhibitory neurotransmitter in the human brain is involved in the regulation of the recovery of fear. Providing insight into the neurochemical basis of fear learning, these findings highlight the opposing role of dACC GABA in the successful extinction of learned fear and its retention over time. Results show that individual differences in dACC GABA predict the propensity for the recovery of fear the following day on both the physiological and the neural level within an interconnected brain circuit in a large sample of healthy individuals. GABA concentrations appear to influence the recovery of fear through modulation of amygdala responsivity, indicating that high baseline GABA levels are associated to increased fear recovery as suggested by both skin conductance responses and BOLD data. Separate analyses of extinction training and the extinction retrieval showed that GABA diminished fear responses to the conditioned stimulus in a large fear network during the late phase of extinction but not during extinction retrieval. No significant association between GABA and physiological responses was observed during these distinct phases, suggesting that GABA primarily affected 


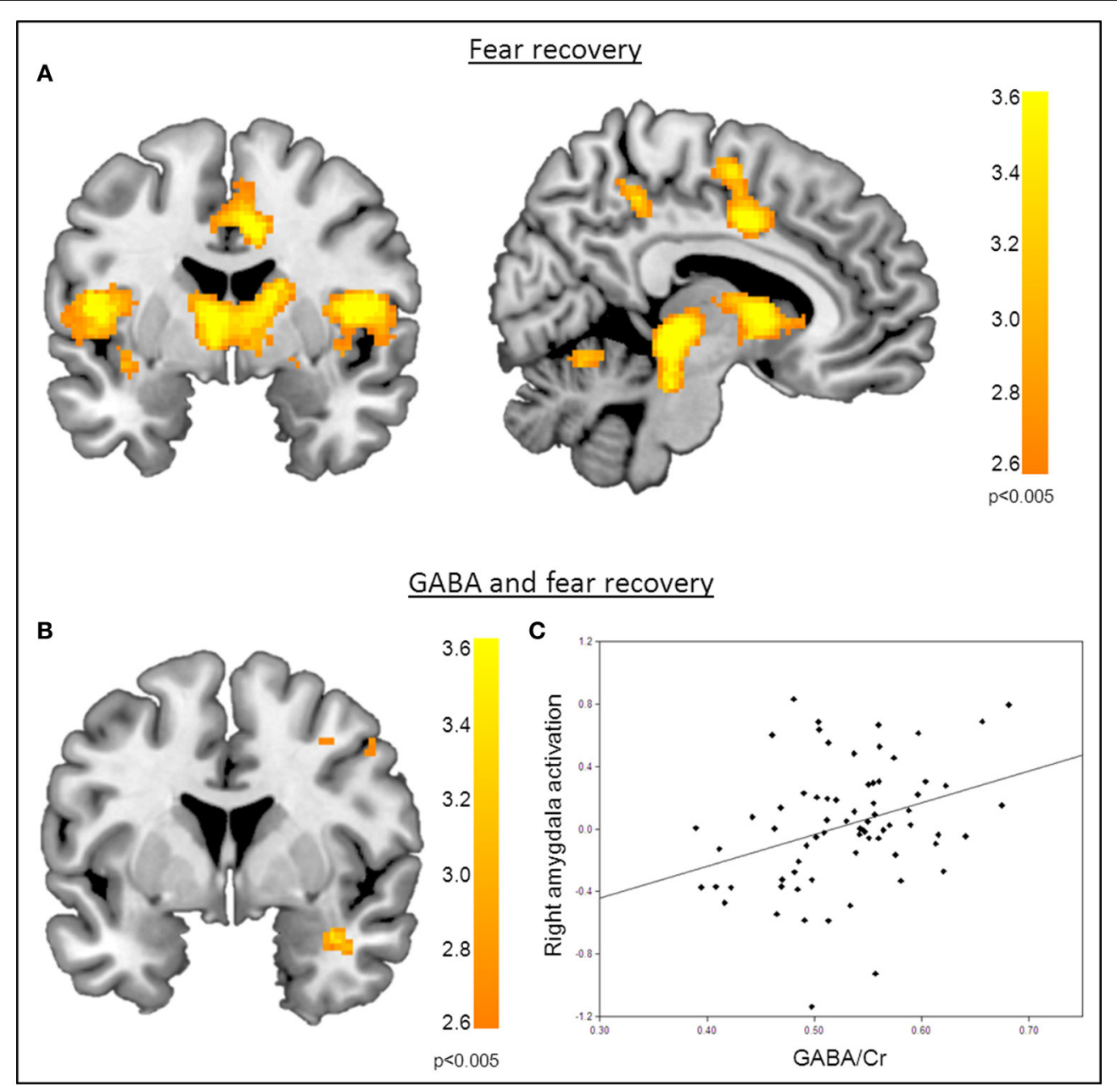

FIGURE 3 | Correlation of GABA levels and BOLD activity during fear recovery. (A) Increased BOLD activity in the middle cingulate gyrus, the thalamus, the right putamen, and the supramarginal gyrus during fear recovery. (B) Voxel-wise correlation of GABA and right amygdala activation during fear recovery, showing that high GABA levels are associated with increased activation of the right amygdala $\left(\mathrm{MNI}_{\mathrm{x}, \mathrm{y}, \mathrm{z}}=36,2,-24 ; \mathrm{P}_{\mathrm{SVC}}<0.05\right)$. Threshold for displaying the images is set at $P=0.005$, uncorrected. (C) Correlation between GABA concentrations and BOLD signal changes extracted from the right amygdala during fear recovery for illustration purposes, indicating that high GABA levels lead to increased fear recovery while low GABA levels are associated with a decrease in fear recovery.

our index of fear recovery that assesses the increase in skin conductance responses from late extinction learning to early extinction retrieval. Although the physiological results suggest that $\mathrm{dACC}$ GABA has no influence on extinction learning or extinction retrieval separately, the neuroimaging results during the extinction training session also suggest that dACC GABA is involved in the inhibition of conditioned fear responses during extinction learning. Together with the fear recovery results, this suggests that dACC GABA is differentially associated with the extinction of fear and its later recovery, and may therefore be particularly important in the consolidation of extinction memory. While our results and study design do not provide any information on causal effects of GABA on functional variability, this interpretation is consistent with findings from rodent studies showing that increasing inhibitory neurotransmission after extinction training interferes with the consolidation of extinction (McGaugh et al., 1990; Akirav, 2007). While the disrupting role of GABA in the consolidation of extinction is well accepted, findings are less consistent for extinction learning. Several studies suggest that increased GABAergic neurotransmission facilitates extinction learning, whereas others have shown no influence of GABA on extinction learning (Harris and Westbrook, 1998; Akirav et al., 2006; Hart et al., 2009; Makkar et al., 2010). However, despite the absence of GABA and psychophysiological correlations, our fMRI findings indicate support for the former, and suggest that GABA has a facilitating role in human extinction learning.

These opposing effects have been observed for both systemic and local interventions. Local interventions mainly focused on three brain regions: the infralimbic cortex, the prelimbic cortex, and the (basolateral) amygdala. Interference of GABAergic neurotransmission in the infralimbic cortex and amygdala have been shown to disrupt extinction consolidation, but the prelimbic cortex has not been found to play a role in extinction 


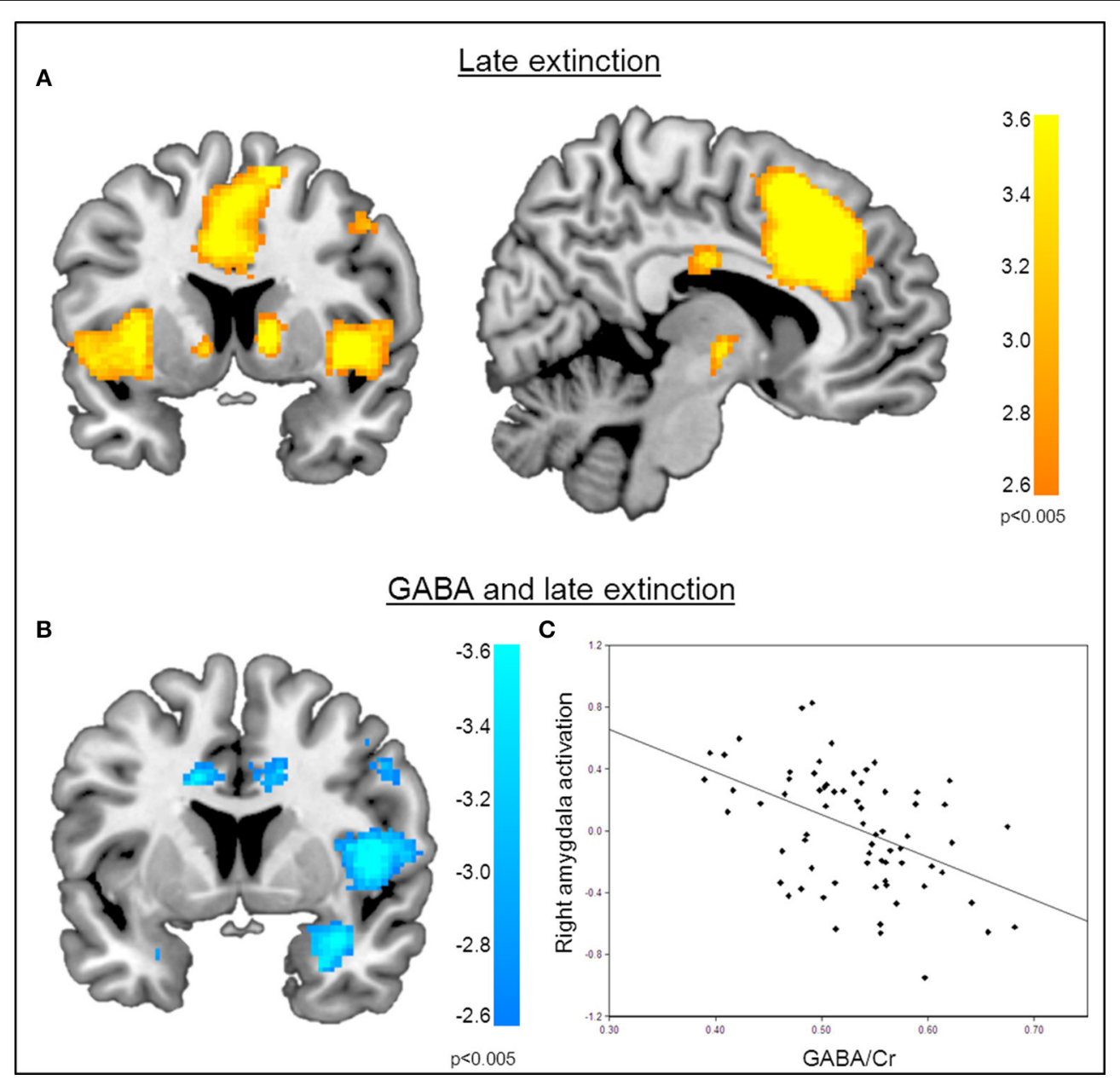

FIGURE 4 | Correlation of GABA levels and BOLD activity during late extinction. (A) Increased BOLD activity in the dACC, the insula, and the superior medial gyrus for the "CS $+>$ CS-" contrast during the late phase of extinction. (B) GABA mediated deactivation of the right amygdala (MNI $I_{x, y, z}=28,4,-28 ; P_{S V C}<0.05$ ), right insula $\left(M N I_{x, y, z}=48,8,6\right.$; $\left.P_{F W E}=0.008\right)$, and middle cingulate gyrus $\left(\left.M_{N}\right|_{x, y, z}=16,-22,34 ; P_{F W E}<0.001\right)$ during the late phase of extinction learning, indicating that high GABA levels are associated with diminished activation of the right amygdala. For displaying purpose, the image threshold is set to $p=0.005$, uncorrected. (C) Correlation plot of GABA levels and activation extracted from the right amygdala during late extinction for illustration purposes, suggesting that high GABA levels facilitate extinction learning while low GABA levels hamper extinction learning.

consolidation in rodents (Akirav, 2007; Laurent and Westbrook, 2009). The prelimbic cortex is assumed to be homologous to the human dACC, but in contrast to rodent studies, our findings do show an inhibitory role of the human dACC in extinction learning and consolidation. A possible interpretation of the opposing association between GABA and the consolidation of extinction memories could be that dACC GABA levels interfere with neuronal activity in the amygdala which would be required to successfully consolidate and express the newly generated memory association (Akirav et al., 2006; VidalGonzalez et al., 2006; Likhtik et al., 2008). This could happen either directly through dACC-amygdala connectivity or through interconnected brain regions of the $\mathrm{dACC}$ and amygdala which may be modulated by dACC GABA levels. One possibility suggested by our results is that this association is mediated by the vmPFC. These findings are in line with previous findings that showed decreased amygdala-vmPFC coupling during memory consolidation after a fear conditioning paradigm (Feng et al., 2014). Functional connectivity data revealed GABA modulated coupling of the amygdala and the vmPFC during the recovery of fear, with high levels of dACC GABA predicting reduced connectivity between these regions, potentially resulting in diminished inhibitory control of the vmPFC on the amygdala. A lack of modulatory control on the amygdala could lead to an increase in amygdala activity, thereby promoting the recovery of fear responses. These findings extend previous evidence by Delli Pizzi et al. (2016b) who found mPFC GABA levels to be inversely related with negative functional coupling of the amygdala and the vmPFC during resting fMRI. In addition, they found trait anxiety to be inversely related to functional amygdala-vmPFC coupling. Similarly to BOLD activity results, the opposite effect of GABAergic abundance on functional connectivity between the 
TABLE 3 | GABA and BOLD responses.

Voxel-wise correlations of dACC GABA and BOLD activation

\begin{tabular}{|c|c|c|c|c|}
\hline Area of activation & $P$-value FWE & Cluster size \# of voxels & $T$ (peak) & Peak voxel MNI coordinates \\
\hline \multicolumn{5}{|c|}{ FEAR RECOVERY AND GABA } \\
\hline Positive correlation & Cluster level & & & \\
\hline Fear network RO/s* & Peak level & & & \\
\hline Right amygdala & $0.022^{\star}$ & 1 & 3.27 & $36,2,-24$ \\
\hline \multicolumn{5}{|c|}{ LATE EXTINCTION AND GABA } \\
\hline Negative correlation & Cluster level & & & \\
\hline Left cerebellum & $<0.0001$ & 719 & 5.07 & $-4,-40,-10$ \\
\hline Right cerebellum & & & 4.03 & $16,-42,-14$ \\
\hline Cerebellum & & & 3.84 & $6,-32,-18$ \\
\hline Right insula lobe & 0.008 & 291 & 4.04 & $48,8,6$ \\
\hline Fear network ROIs* & Peak level & & & \\
\hline Right amygdala & $0.012^{\star}$ & 7 & 3.46 & $28,4,-28$ \\
\hline
\end{tabular}

GABA mediated psychophysiological interactions using a right amygdala seed

\section{FEAR RECOVERY AND GABA}

Negative correlation

Fear network RO/s*

$0.023^{*}$

$\begin{array}{ll}0.023^{*} & 47\end{array}$

3.51

$10,52,-2$

LATE EXTINCTION AND GABA

Positive correlation

Fear network RO/s* Peak level

$\begin{array}{llrl}\text { vmPFC } & 0.003^{*} & 219 & 0,44,-2\end{array}$

All results are $P<0.05$ cluster-level FWE corrected with an initial height threshold of $P=0.001$, uncorrected.

*Small volume corrected with $P_{\text {peak voxel }}<0.05$.

amygdala and the vmPFC was observed during fear extinction. Enhanced coupling of the amygdala and the vmPFC was shown to be mediated by high levels of GABA, suggesting that this increase in connectivity facilitates prefrontal control of the amygdala, thereby facilitating the extinction of conditioned fear.

In the light of interpreting our findings, it is important to highlight that the main aim of this study is to investigate factors that contribute to individual differences in human fear recovery, rather than providing a mechanistic account of how GABA regulates fear learning as it is beyond the scope of this study and the available techniques to investigate the fine-grained fear circuitry identified in animal studies. With regards to our GABA measurements, it is important to point to the lack of clarity with regards to the interpretation of the GABA MRS signal source and how it relates to synaptic activity (Stagg et al., 2011; Rae, 2014). Potential sources can be cytoplasmic, vesicular, and free extracellular GABA with metabolic, neurotransmission, and neuromodulatory functions, respectively. Both vesicular and free extracellular concentrations have been suggested to be represented in the GABA MRS signal. However, a clear distinction between GABA pools is not possible and remains subject to discussion (Martin and Rimvall, 1993; Belelli et al., 2009). Furthermore, the baseline measurement of GABA levels on the first experimental day and the interpretation of our results assume the stability of GABA levels in the dorsal ACC over longer periods of time. Although prefrontal GABA levels have been shown to drop during acute stress (Hasler et al., 2010), resting GABA measurements have shown good reproducibility for within-day measurements as well as for an interval of 7 months (Evans et al., 2010; Near et al., 2014). Individual differences in GABA levels should therefore remain stable over the $24 \mathrm{~h}$ time period between sessions. Even though the aim of this study was to examine correlations of baseline GABA levels and fear recovery, investigating changes in GABA levels throughout the experiment would provide further important insights into role of GABA on fear responses. Future studies should aim at assessing GABA levels at different time points throughout the fear learning paradigm and how this relates to baseline GABA concentrations. Another potential limitation of the study is the high number of excluded spectra. For specificity of voxel location and voxel dimensions, we aimed for precise coverage of the region of expected dACC BOLD activity during fear conditioning. The resulting voxel volume of $16 \mathrm{~cm}^{3}$ is relatively small for GABAedited MRS and resulted in a substantial number of spectra that were of insufficient quality.

These results provide a first step to understand how differences in brain GABA concentrations may relate to fear related disorders. Our results show that healthy individuals with high GABA levels show higher fear recovery. 


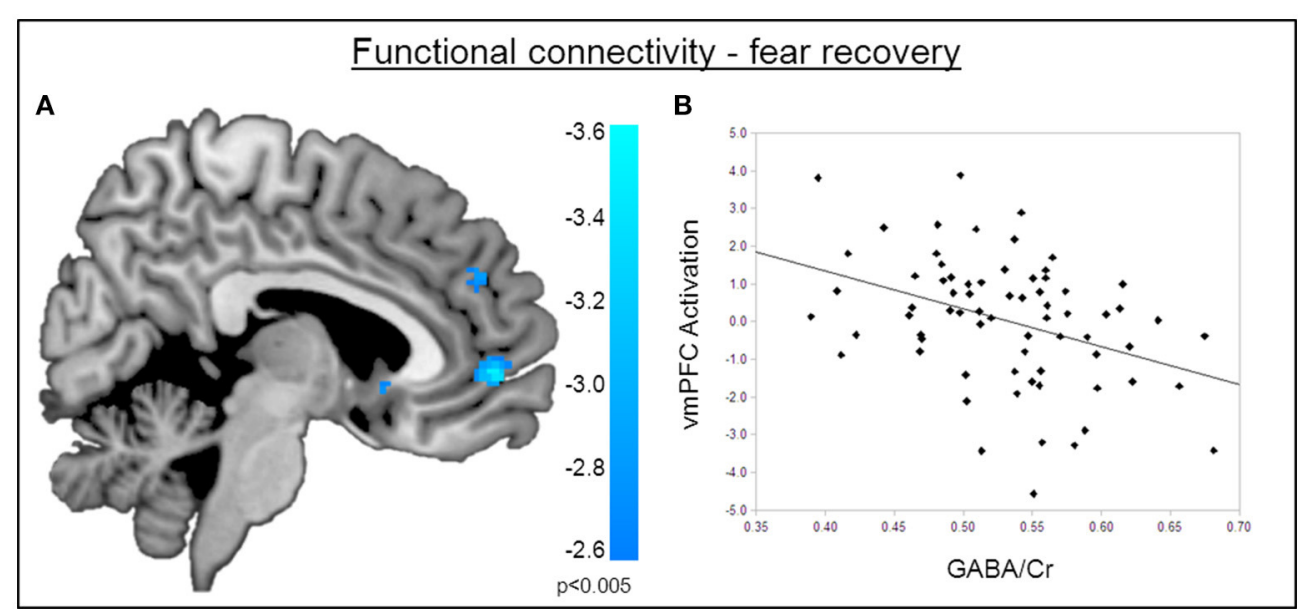

FIGURE 5 | Functional connectivity during the recovery of fear. (A) Psychophysiological interactions with the right amygdala seed region during fear recovery revealed a negative voxel-wise correlation of GABA mediated amygdala-vmPFC connectivity during fear recovery ( $\left.\mathrm{MNI}_{x, y, z}=2,52,-2 ; \mathrm{P}_{S V C}<0.05\right)$. Threshold for displaying the images is set at $P=0.005$, uncorrected. (B) Correlation plot of dACC GABA levels and deactivation of the vmPFC showing that high GABA levels are correlated with diminished amygdala-vmPFC connectivity compared to low GABA levels.

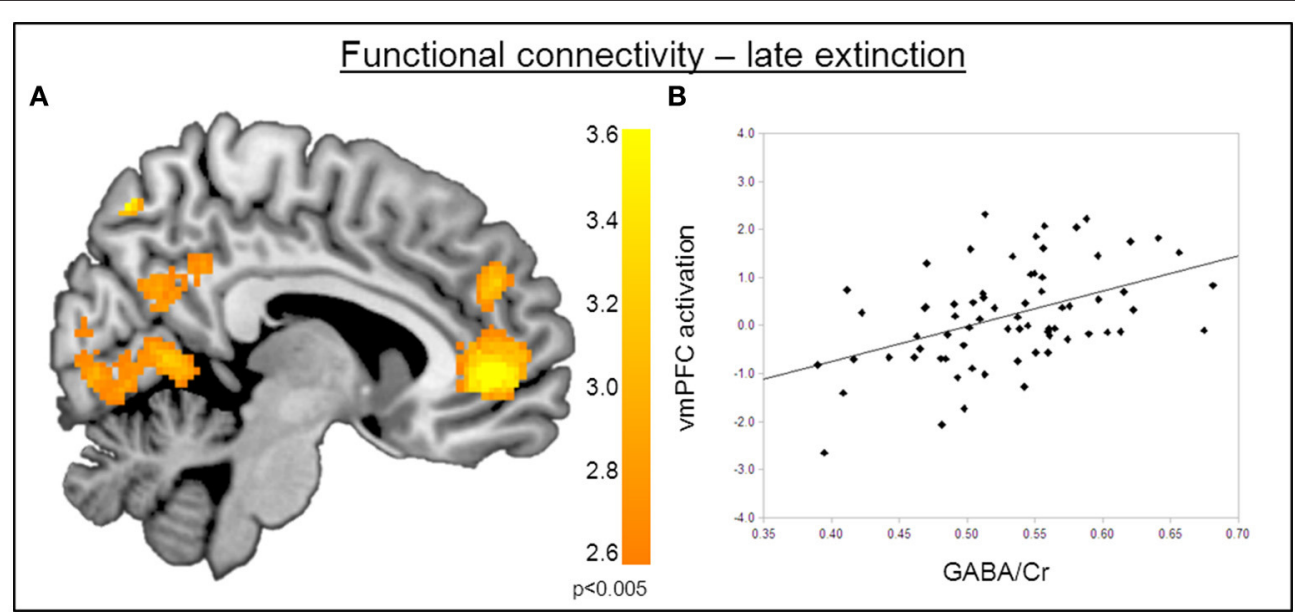

FIGURE 6 | Functional connectivity during late extinction. (A) A psychophysiological interaction analysis revealed that high levels of GABA mediate an increase in connectivity between the amygdala seed region and the vmPFC ( $\left.M N I_{x, y, z}=-4,-40,-10\right)$ during extinction ( $\left.P_{F W E}<0.05\right)$. (B) Correlation plot illustrating a positive relation between dACC GABA levels and amygdala-vmPFC connectivity.

A recent study in patients with PTSD showed that ACC GABA concentrations are significantly higher in patients compared to healthy controls (Michels et al., 2014). In addition, the administration of a GABA agonist acutely reduces anxiety but also reduce the efficacy of exposure therapy in PTSD (Rothbaum et al., 2014). Both studies point to increased GABA concentrations and GABAergic neurotransmission as a critical aspect in PTSD. Our results suggest that increased GABAergic activity reduces the consolidation of extinction training and leads to more fear recovery, which may explain the high levels of fear in PTSD patients and adverse effects of GABA agonists on long-term outcome.
In conclusion, these results show that individual differences in dACC GABA levels are associated with differences in the intensity of fear recovery in healthy young men. We suggest that this might potentially be through differential modulation of activity and connectivity within a tightly interconnected network involving the amygdala, dACC, and vmPFC. Our findings particularly highlight that correlations between GABA and both neural and psychophysiological fear measures differ not only depending on neurotransmitter quantity but are also dependent on the phase of fear learning, partially revealing opposing effects during fear recovery and extinction learning. While high GABA concentrations appear to benefit extinction learning, associations with the 
consolidation of extinction point into a negative direction. Our findings are intriguing as they link findings from rodents to humans which should be further extended to anxiety related disorders.

\section{AUTHOR CONTRIBUTIONS}

NL and GAvW designed research; NL and JvL performed research; NL analyzed data; NL and GAvW wrote the first draft; JvL, NP, DD contributed to and approved the manuscript.

\section{REFERENCES}

Akirav, I. (2007). NMDA Partial agonist reverses blocking of extinction of aversive memory by GABA(A) agonist in the amygdala. Neuropsychopharmacology 32, 542-550. doi: 10.1038/sj.npp.1301050

Akirav, I., Raizel, H., and Maroun, M. (2006). Enhancement of conditioned fear extinction by infusion of the GABA A agonist muscimol into the rat prefrontal cortex and amygdala. Eur. J. Neurosci. 23, 758-764. doi: $10.1111 / j .1460-9568.2006 .04603 . x$

Bach, D. R., Weiskopf, N., and Dolan, R. J. (2011). A stable sparse fear memory trace in human amygdala. J. Neurosci. 31, 9383-9389. doi: 10.1523/JNEUROSCI.1524-11.2011

Belelli, D., Harrison, N. L., Maguire, J., Macdonald, R. L., Walker, M. C., and Cope, D. W. (2009). Extrasynaptic GABAA receptors: form, pharmacology, and function. J. Neurosci. 29, 12757-12763. doi: 10.1523/JNEUROSCI.3340-09.2009

Bindra, D., Nyman, K., and Wise, J. (1965). Barbiturate-induced dissociation of acquisition and extinction: role of movement-initiating processes. J. Comp. Physiol. Psychol. 60, 223-228. doi: 10.1037/h0022327

Bouton, M. E. (2004). Context and behavioral processes in extinction. Learn. Mem. 11, 485-494. doi: 10.1101/lm.78804

Bouton, M. E., Kenney, F. A., and Rosengard, C. (1990). State-dependent fear extinction with two benzodiazepine tranquilizers. Behav. Neurosci. 104, 44-55. doi: 10.1037/0735-7044.104.1.44

Bush, D. E., Sotres-Bayon, F., and Ledoux, J. E. (2007). Individual differences in fear: isolating fear reactivity and fear recovery phenotypes. J. Trauma. Stress 20 , 413-422. doi: 10.1002/jts.20261

Delli Pizzi, S., Chiacchiaretta, P., Mantini, D., Bubbico, G., Ferretti, A., Edden, R. A., et al. (2016a). Functional and neurochemical interactions within the amygdala-medial prefrontal cortex circuit and their relevance to emotional processing. Brain Struct. Funct. 222, 1267-1279. doi: 10.1007/s00429-016-1276-Z

Delli Pizzi, S., Padulo, C., Brancucci, A., Bubbico, G., Edden, R. A., Ferretti, A., et al. (2016b). GABA content within the ventromedial prefrontal cortex is related to trait anxiety. Soc. Cogn. Affect. Neurosci. 11, 758-766. doi: 10.1093/scan/nsv155

Delorme, A., and Makeig, S. (2004). EEGLAB: an open source toolbox for analysis of single-trial EEG dynamics including independent component analysis. $J$. Neurosci. Methods 134, 9-21. doi: 10.1016/j.jneumeth.2003.10.009

Duits, P., Cath, D. C., Lissek, S., Hox, J. J., Hamm, A. O., Engelhard, I. M., et al. (2015). Updated meta-analysis of classical fear conditioning in the anxiety disorders. Depress. Anxiety 32, 239-253. doi: 10.1002/da.22353

Durham, R. C., Chambers, J. A., Power, K. G., Sharp, D. M., Macdonald, R. R., Major, K. A., et al. (2005). Long-term outcome of cognitive behaviour therapy clinical trials in central Scotland. Health Technol. Assess. 9, 1-128. doi: $10.3310 /$ hta9420

Eklund, A., Nichols, T. E., and Knutsson, H. (2016). Cluster failure: why fMRI inferences for spatial extent have inflated false-positive rates. Proc. Natl. Acad. Sci. U.S.A. 113, 7900-7905. doi: 10.1073/pnas.1602413113

Etkin, A., Egner, T., and Kalisch, R. (2011). Emotional processing in anterior cingulate and medial prefrontal. Trends Cogn. Sci. 15, 85-93. doi: 10.1016/j.tics.2010.11.004

Evans, C. J., McGonigle, D. J., and Edden, R. A. (2010). Diurnal stability of gamma-aminobutyric acid concentration in visual and sensorimotor

\section{FUNDING}

This work was supported by the Netherlands Organization for Scientific Research (NWO/ZonMW 916.11.037) and by the AMC Reseach Council (110913).

\section{SUPPLEMENTARY MATERIAL}

The Supplementary Material for this article can be found online at: http://journal.frontiersin.org/article/10.3389/fnhum. 2017.00202/full\#supplementary-material

cortex. J. Magn. Reson. Imaging 31, 204-209. doi: 10.1002/jmri. 21996

Feng, P., Feng, T., Chen, Z., and Lei, X. (2014). Memory consolidation of fear conditioning: bi-stable amygdala connectivity with dorsal anterior cingulate and medial prefrontal cortex. Soc. Cogn. Affect. Neurosci. 9, 1730-1737. doi: $10.1093 /$ scan/nst170

Ghashghaei, H. T., Hilgetag, C. C., and Barbas, H. (2007). Sequence of information processing for emotions based on the anatomic dialogue between prefrontal cortex and amygdala. Neuroimage 34, 905-923. doi: 10.1016/j.neuroimage.2006.09.046

Harris, J. A., and Westbrook, R. F. (1998). Evidence that GABA transmission mediates context-specific extinction of learned fear. Psychopharmacology (Berl) 140, 105-115. doi: 10.1007/s002130050745

Hart, G., Harris, J. A., and Westbrook, R. F. (2009). Systemic or intraamygdala injection of a benzodiazepine (midazolam) impairs extinction but spares re-extinction of conditioned fear responses. Learn. Mem. 16, 53-61. doi: 10.1101/lm.1154409

Hasler, G., van der Veen, J. W., Grillon, C., Drevets, W. C., and Shen, J. (2010). Effect of acute psychological stress on prefrontal. Am. J. Psychiatry 167, 1226-1231. doi: 10.1176/appi.ajp.2010.09070994

Klavir, O., Genud-Gabai, R., and Paz, R. (2013). Functional connectivity between amygdala and cingulate cortex for adaptive aversive learning. Neuron 80, 1290-1300. doi: 10.1016/j.neuron.2013.09.035

Laurent, V., and Westbrook, R. F. (2009). Inactivation of the infralimbic but not the prelimbic cortex impairs consolidation and retrieval of fear extinction. Learn. Mem. 16, 520-529. doi: 10.1101/lm.1474609

Likhtik, E., Popa, D., Apergis-Schoute, J., Fidacaro, G. A, and Paré, D. (2008). Amygdala intercalated neurons are required for expression of fear extinction. Nature 454, 642-645. doi: 10.1038/nature07167

MacNamara, A., Rabinak, C. A., Fitzgerald, D. A., Zhou, X. J., Shankman, S. A., Milad, M. R., et al. (2015). Neural correlates of individual differences in fear learning. Behav. Brain Res. 287, 34-41. doi: 10.1016/j.bbr.2015. 03.035

Makkar, S. R., Zhang, S. Q., and Cranney, J. (2010). Behavioral and neural analysis of GABA in the acquisition, consolidation, reconsolidation, and extinction of fear memory. Neuropsychopharmacology 35, 1625-1652. doi: 10.1038/npp.2010.53

Martin, D. L., and Rimvall, K. (1993). Regulation of gammaaminobutyric acid synthesis in the brain. J. Neurochem. 60, 395-407. doi: 10.1111/j.1471-4159.1993.tb03165.x

McGaugh, J. L., Introini-Collison, I. B., Nagahara, A. H., Cahill, L., Brioni, J. D., and Castellano, C. (1990). Involvement of the amygdaloid complex in neuromodulatory influences on memory storage. Neurosci. Biobehav. Rev. 14, 425-431. doi: 10.1016/S0149-7634(05)80065-X

McLaren, D. G., Ries, M. L., Xu, G., and Johnson, S. C. (2012). A generalized form of context-dependent psychophysiological interactions (gPPI): a comparison to standard approaches. Neuroimage 61, 1277-1286. doi: 10.1016/j.neuroimage.2012.03.068

Mescher, M., Merkle, H., Kirsch, J., Garwood, M., and Gruetter, R. (1998). Simultaneous in vivo spectral editing and water suppression. NMR Biomed. 11, 266-272. doi: 10.1002/(SICI)1099-1492(199810)11:6<266::AID-NBM530>3.0. $\mathrm{CO} ; 2-\mathrm{J}$ 
Michels, L., Schulte-vels, T., Schick, M., O’Gorman, R. L., Zef, T., Hasler, G., et al. (2014). Prefrontal GABA and glutathione imbalance in posttraumatic stress disorder: preliminary findings. Psychiatry Res. 224, 288-295. doi: 10.1016/j.pscychresns.2014.09.007

Milad, M. R., Furtak, S. C., Greenberg, J. L., Keshaviah, A., Im, J. J., Falkenstein, M. J., et al. (2013). Deficits in conditioned fear extinction in obsessive-compulsive disorder and neurobiological changes in the fear circuit. JAMA Psychiatry 70, 608-618. doi: 10.1001/jamapsychiatry.2013.914

Milad, M. R., Orr, S. P., Lasko, N. B., Chang, Y., Rauch, S. L., and Pitman, R. K. (2008). Presence and acquired origin of reduced recall for fear extinction in PTSD: results of a twin study. J. Psychiatr. Res. 42, 515-520. doi: 10.1016/j.jpsychires.2008.01.017

Milad, M. R., and Quirk, G. J. (2002). Neurons in medial prefrontal cortex signal memory for fear extinction. Nature 420, 70-74. doi: 10.1038/nature01138

Milad, M. R., Wright, C. I., Orr, S. P., Pitman, R. K., Quirk, G. J., and Rauch, S. L. (2007). Recall of fear extinction in humans activates the ventromedial prefrontal cortex and hippocampus in concert. Biol. Psychiatry 62, 446-454. doi: 10.1016/j.biopsych.2006.10.011

Motzkin, J. C., Philippi, C. L., Wolf, R. C., Baskaya, M. K., and Koenigs, M. (2015). Ventromedial prefrontal cortex is critical for the regulation of amygdala activity in humans. Biol. Psychiatry 77, 276-284. doi: 10.1016/j.biopsych.2014.02.014

Muthukumaraswamy, S. D., Edden, R. A., Jones, D. K., Swettenham, J. B., and Singh, K. D. (2009). Resting GABA concentration predicts peak gamma frequency and fMRI amplitude in response to visual stimulation in humans. Proc. Natl. Acad. Sci. U.S.A. 106, 8356-8361. doi: 10.1073/pnas.0900728106

Naressi, A., Couturier, C., Castang, I., de Beer, R., and Graveron-Demilly, D. (2001). Java-based graphical user interface for MRUI, a software package for quantitation of in vivo/medical magnetic resonance spectroscopy signals. Comput. Biol. Med. 31, 269-286. doi: 10.1016/S0010-4825(01)00006-3

Near, J., Ho, Y. C. L., Sandberg, K., Kumaragamage, C., and Blicher, J. U. (2014). Long-term reproducibility of GABA magnetic resonance spectroscopy. Neuroimage 99, 191-196. doi: 10.1016/j.neuroimage.2014.05.059

Northoff, G., Walter, M., Schulte, R. F., Beck, J., Dydak, U., Henning, A., et al. (2007). GABA concentrations in the human anterior cingulate cortex predict negative BOLD responses in fMRI. Nat. Neurosci. 10, 1515-1517. doi: $10.1038 / \mathrm{nn} 2001$

Pavlov, P. I. (1927). Conditioned reflexes: an investigation of the physiological activity of the cerebral cortex. Ann. Neurosci. 17, 136-141. doi: 10.2307/1134737

Phelps, E. A., Delgado, M. R., Nearing, K. I., and Ledoux, J. E. (2004). Extinction learning in humans: role of the amygdala and vmPFC. Neuron 43, 897-905. doi: 10.1016/j.neuron.2004.08.042

Quirk, G. J., Likhtik, E., Pelletier, J. G., and Paré, D. (2003). Stimulation of medial prefrontal cortex decreases the responsiveness of central amygdala output neurons. J. Neurosci. 23, 8800-8807. doi: 10.1016/j.neuron.2004.08.042

Rae, C. D. (2014). A guide to the metabolic pathways and function of metabolites observed in human brain $1 \mathrm{H}$ magnetic resonance spectra. Neurochem. Res. 39, 1-36. doi: 10.1007/s11064-013-1199-5

Rescorla, R. A. (2004). Spontaneous recovery varies inversely with the trainingextinction interval. Learn. Behav. 32, 401-408. doi: 10.3758/BF03196037

Rosenkranz, J. A., Moore, H., and Grace, A. A. (2003). The prefrontal cortex regulates lateral amygdala neuronal plasticity and responses to previously conditioned stimuli. J. Neurosci. 23, 11054-11064.
Rothbaum, B. O., Price, M., Jovanovic, T., Norrholm, S. D., Gerardi, M., Dunlop, B., et al. (2014). A randomized, double-blind evaluation of D-cycloserine or alprazolam combined with virtual reality exposure therapy for posttraumatic stress disorder in Iraq and Afghanistan War veterans. Am. J. Psychiatry 171, 640-648. doi: 10.1176/appi.ajp.2014.13121625

Sehlmeyer, C., Schöning, S., Zwitserlood, P., Pfleiderer, B., Kircher, T., Arolt, V., et al. (2009). Human fear conditioning and extinction in neuroimaging: a systematic review. PLOS ONE 4:e5865. doi: 10.1371/journal.pone. 0005865

Shin, L. M., and Liberzon, I. (2010). The neurocircuitry of fear, stress, and anxiety disorders. Neuropsychopharmacology 35, 169-191. doi: 10.1038/npp.2009.83

Stagg, C. J., Bachtiar, V., and Johansen-Berg, H. (2011). What are we measuring with GABA magnetic resonance spectroscopy? Commun. Integr. Biol. 4, 573-575. doi: $10.4161 /$ cib. 16213

Sumner, P., Edden, R. A. E., Bompas, A., Evans, C. J., and Singh, K. D. (2010). More GABA, less distraction: a neurochemical predictor of motor decision speed. Nat. Neurosci. 13, 825-827. doi: 10.1038/nn.2559

Tzourio-Mazoyer, N., Landeau, B., Papathanassiou, D., Crivello, F., Etard, O., Delcroix, N., et al. (2002). Automated anatomical labeling of activations in SPM using a macroscopic anatomical parcellation of the MNI MRI single-subject brain. Neuroimage 15, 273-289. doi: 10.1006/nimg.2001.0978

Vidal-Gonzalez, I., Vidal-Gonzalez, B., Rauch, S. L., and Quirk, G. J. (2006). Microstimulation reveals opposing influences of prelimbic and infralimbic cortex on the expression of conditioned fear. Learn. Mem. 13, 728-733. doi: 10.1101/lm.306106

Waddell, K. W., Avison, M. J., Joers, J. M., and Gore, J. C. (2007). A practical guide to robust detection of GABA in human brain by J-difference spectroscopy at $3 \mathrm{~T}$ using a standard volume coil. Magn. Reson. Imaging 25, 1032-1038. doi: 10.1016/j.mri.2006.11.026

Worsley, K. J., Marrett, S., Neelin, P., Vandal, A. C., Friston, K. J., and Evans, A. C. (1996). A unified statistical approach for determining significant signals in images of cerebral activation. Hum. Brain Mapp. 4, 58-73. doi: 10.1002/ (SICI)1097-0193(1996)4:1<58::AID-HBM4>3.0.CO;2-O

Conflict of Interest Statement: DD is a member of the advisory board of Lundbeck. He receives occasional fees from Medtronic for educational purposes.

The reviewer MT and handling Editor declared their shared affiliation, and the handling Editor states that the process nevertheless met the standards of a fair and objective review.

The other authors declare that the research was conducted in the absence of any commercial or financial relationships that could be construed as a potential conflict of interest.

Copyright (C) 2017 Levar, van Leeuwen, Puts, Denys and van Wingen. This is an open-access article distributed under the terms of the Creative Commons Attribution License (CC BY). The use, distribution or reproduction in other forums is permitted, provided the original author(s) or licensor are credited and that the original publication in this journal is cited, in accordance with accepted academic practice. No use, distribution or reproduction is permitted which does not comply with these terms. 